EPJ Web of Conferences 60, 20003 (2013)

DOI: $10.1051 /$ epjconf $/ 20136020003$

(C) Owned by the authors, published by EDP Sciences, 2013

\title{
Diffraction studies with ALICE
}

\author{
Pedro González Zamora ${ }^{1, a}$ (for the ALICE Collaboration) \\ ${ }^{1}$ Centro de Investigaciones Energéticas, Medioambientales y Técnologicas (CIEMAT), Madrid, Spain.
}

\begin{abstract}
The measurement of Single and Double Diffraction cross-sections in pp collisions by the ALICE Collaboration at $\sqrt{s}=0.9,2.76$ and $7 \mathrm{TeV}$ will be presented. The relevance of diffraction to the understanding of inelastic pp interactions will be discussed and the measurement of the inelastic pp cross-section will be presented. A brief status of ALICE's studies of centrally produced systems, selected with a two-pseudorapidity gap topology, will also be given.
\end{abstract}

\section{Introduction}

The cross-sections of inelastic and diffractive processes in proton-proton (pp) collisions are among the basic obervables used to characterize the global properties of interactions. Two contributions to the inelastic cross-section are diffractive processes and non-diffractive processes. Regge theory is the main framework for describing diffractive processes. Diffraction proceeds via the exchange of Pomerons. Experimentally, it is not possible to distinguish large rapidity gaps caused by Pomeron exchange from those caused by other colour-neutral exchanges. The separation of these processes is model dependent. In order to provide the dependence of Single Diffraction (SD) crosssection on diffracted mass, PYTHIA6 and PHOJET were modified to use the model [1]. ALICE measured inelastic, single- and double-diffraction cross-sections in pp collisions [2].

\section{The ALICE Experiment}

The ALICE Experiment [3] at the Large Hadron Collider (LHC) consists mainly of the central barrel in the pseudorapidity range $-0.9<\eta<0.9$ and a muon spectrometer at $-2.5<\eta<-4.0$. The analysis presented here is based on the data from the VZERO detector, the Silicon Pixel Detector (SPD) and the Forward Multiplicity Detector (FMD). Combining VZERO, SPD and FMD, ALICE has a continuous acceptance over a pseudorapidity interval of $-3.7<\eta<5.1$ (8.8 units). The SPD and VZERO are the main ALICE triggers for the selection of minimum bias events. The Inner Tracking System (ITS) and the Time-Projection Chamber (TPC) are used only for vertex reconstruction in the cross-section analysis and for vertex reconstruction and tracking in the central diffraction analysis.

\footnotetext{
a. e-mail: pedro.gonzalez@externos.ciemat.es
}

\section{Measurement of cross-sections}

\subsection{Relative rate of single and double diffraction}

A study of gaps in the pseudorapidity distribution of particles was carried out in order to determine the fractions of diffractive processes in inelastic collisions. The pseudorapidity distribution of tracks consisting of the event vertex and a hit in either SPD, VZERO and FMD cells was measured on an event-by-event basis. Afterwards, the events were classified into three categories, called "one-arm-L", "one-arm-R", and "two-arm" (a detailed description of the classification can be found in Ref. [2]). The intention of this was to increase the sensitivity to diffractive processes. The categories "one-arm-L" and "one-arm-R" have an enriched SD component, while a subset of the two-arm category can be associated with Double Diffraction (DD).

The fraction of SD processes was measured by counting the relative rate of one-arm-L(R) and two-arm classes. In MC simulations, it was observed that masses above 200 $\mathrm{GeV} / c^{2}$ are mainly classified as two-arm at all three energies, therefore $M_{X}=200 \mathrm{GeV} / c^{2}$ was chosen as the boundary between SD and non-single diffractive (NSD) events. The material budget was varied in the simulation by $\pm 10 \%$ everywhere and by $+50 \%$ in the forward region only $(\eta>1)$. In both cases this did not modify the gap characteristics significantly. A comparison with data showed that with the default DD fractions PYTHIA6 significantly overestimates the fraction of large pseudorapidity gaps and PHOJET significantly underestimates it. In order to estimate the fraction of DD processes, an adjusment was made to the DD fraction in PYTHIA6 and PHOJET using the measured gap width distribution. The mean value between the tuned PYTHIA6 and PHOJET estimates is taken as the best estimate for the DD fraction. The relative rates of SD and DD are shown in Table 1. Despite the different acceptances of the two ALICE sides, results for $\mathrm{SD}$ are symmetrical as expected from the symmetry of the physics process. The SD fraction obtained at $\sqrt{s}=0.9 \mathrm{TeV}$ 
Table 1. Fractions of SD $\left(M<200 \mathrm{GeV} / c^{2}\right)$ and DD $(\Delta \eta>3)$ events. Data are taken from [2].

\begin{tabular}{lllll}
\hline$\sqrt{s}(\mathrm{TeV})$ & $\sigma_{\mathrm{SD}}^{\text {right }} / \sigma_{\text {INEL }}$ & $\sigma_{\mathrm{SD}}^{\text {left }} / \sigma_{\text {INEL }}$ & $\sigma_{\mathrm{SD}} / \sigma_{\text {INEL }}$ & $\sigma_{\mathrm{DD}} / \sigma_{\text {INEL }}$ \\
\hline 0.9 & $0.11 \pm 0.02$ & $0.10 \pm 0.02$ & $0.21 \pm 0.03$ & $0.11 \pm 0.03$ \\
2.76 & $0.11_{-0.05}^{+0.04}$ & $0.09 \pm 0.03$ & $0.20_{-0.08}^{+0.07}$ & $0.12 \pm 0.05$ \\
7 & $0.10_{-0.03}^{+0.02}$ & $0.10_{-0.04}^{+0.02}$ & $0.20_{-0.07}^{+0.04}$ & $0.12_{-0.04}^{+0.05}$ \\
\hline
\end{tabular}

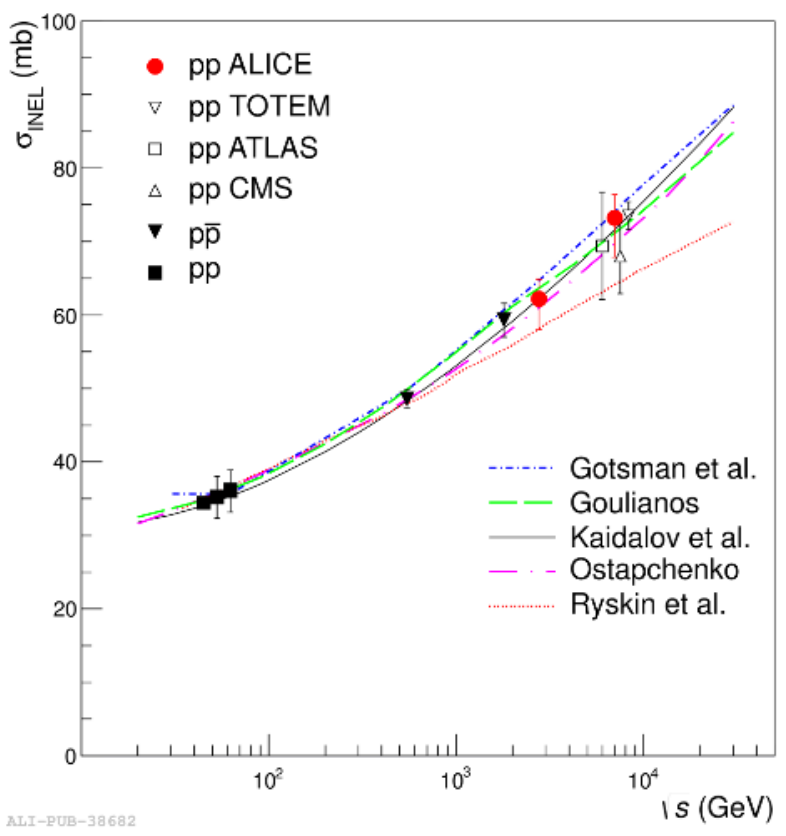

Figure 1. Inelastic cross-sections as a function of centre-of-mass energy, in proton-proton or proton-antiproton collisions, compared with predictions [10] (short-dot-dashed blue line), [11] (dashed green line), [1] (solid black line), [12] (long dot-dashed pink line) and [13] (dotted red line). Plot taken from [2].

is consistent with the UA5 measurement for $\mathrm{p} \overline{\mathrm{p}}$ collisions [8].

\subsection{Inelastic cross-sections}

The inelastic cross-section was measured using the measurement of $A \times \sigma_{\text {INEL }}$ obtained from van der Meer scans alongside a simulation of diffraction adjusted to data. The factor $A$ represents the acceptance and efficiency of the $\mathrm{MB}_{\mathrm{AND}}$ (a coincidence requiring at least one hit in each of the two VZERO arrays). The measured inelastic pp cross sections are:

- $\sigma_{\text {INEL }}=62.8_{-4.0}^{+2.4}($ model $) \pm 1.2($ lumi $) \mathrm{mb}$ at $\sqrt{s}=2.76$ $\mathrm{TeV}$

- $\sigma_{\text {INEL }}=73.2_{-4.6}^{+2.0}($ model $) \pm 2.6($ lumi $) \mathrm{mb}$ at $\sqrt{s}=7 \mathrm{TeV}$

The result at $\sqrt{s}=7 \mathrm{TeV}$ is consistent with measurements by ATLAS [4] and CMS [5], and is very close to the TOTEM [6] result. A comparison of the ALICE results with other measurements at different energies and with model calculations is shown in Figure 1.

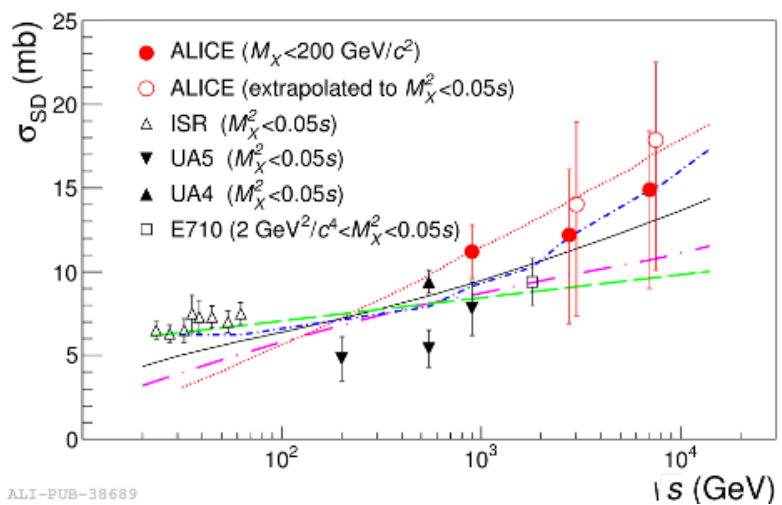

Figure 2. Single-diffractive cross-sections as a function of centre-of-mass energy. The predictions of theoretical models correspond to $M_{X}^{2}<0.05 s$ and are defined as in Figure 1. Plot taken from [2].

Table 2. Inelastic cross-section $\left(\sigma_{\mathrm{INEL}}\right)$ for pp collisions at $\sqrt{s}=$ $7 \mathrm{TeV}$ at the LHC. Data are taken from ALICE [2], ATLAS [4], CMS [5] and TOTEM [6].

\begin{tabular}{ll}
\hline Experiment & $\sigma_{\text {INEL }}(\mathrm{mb})$ \\
\hline ALICE & $73.2_{-4.6}^{+2.0}($ model $) \pm 2.6($ lumi $)$ \\
ATLAS & $69.4 \pm 6.9($ model $) \pm 2.4(\exp )$ \\
CMS & $68.0 \pm 4.0($ model $) \pm 2.0($ syst $) \pm 2.4($ lumi $)$ \\
TOTEM & $73.5_{-1.3}^{+1.8}($ syst $) \pm 0.6($ stat $)$ \\
\hline
\end{tabular}

\subsection{Diffractive cross-sections}

The cross-sections for SD and DD processes shown in Table 3 were obtained by combining the measurements of inelastic cross-sections with the relative rates of diffractive processes. As the inelastic cross-section at $\sqrt{s}=0.9 \mathrm{TeV}$ was not measured by ALICE, the value $\sigma_{\text {INEL }}=52.5_{-3.3}^{+2.0}$ which was derived from UA5 measurement [9] was used. A comparison of ALICE diffraction cross-section measurements with data at previous colliders and with models is shown in Figures 2 and 3.

\section{Central diffraction}

A double gap topology is used as a filter for centraldiffractive events. This topology is defined by activity in the ALICE central barrel and no activity outside. The gaps are defined by the absence of activity somewhere within the trigger signals of VZERO, FMD and SPD in the pseudorapidity ranges of $-3.7<\eta<-0.9\left(\Delta \eta_{1}=2.8\right)$ and 
Table 3. Proton-proton diffractive cross-sections measured by ALICE at $\sqrt{s}=0.9,2.76$ and $7 \mathrm{TeV}$. Single diffraction is for $M_{X}<200 \mathrm{GeV} / c^{2}$ and double diffraction is for $\Delta \eta>3$. Data are taken from [2].

\begin{tabular}{lll}
\hline$\sqrt{s}$ & $\sigma_{\mathrm{SD}}(\mathrm{mb})$ & $\sigma_{\mathrm{DD}}(\mathrm{mb})$ \\
\hline 0.9 & $11.2_{-2.1}^{+1.6}($ syst $)$ & $5.6 \pm 2.0($ syst $)$ \\
2.76 & $12.2_{-5.3}^{+3.9}($ syst $) \pm 0.2$ (lumi) & $7.8 \pm 3.2$ (syst) \pm 0.2 (lumi) \\
7 & $14.9_{-5.9}^{+3.4}$ (syst) \pm 0.5 (lumi) & $9.0 \pm 2.6$ (syst) \pm 0.3 (lumi)
\end{tabular}

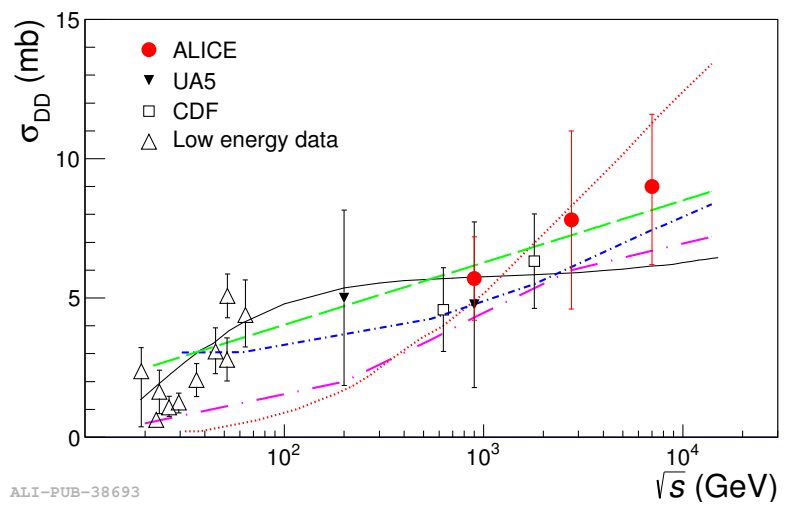

Figure 3. Double-diffractive cross-sections as a function of centre-of-mass energy. The theoretical model predictions represented as lines are for $\Delta \eta>3$ and are defined as Figure 1. Plot taken from [2].

$0.9<\eta<5.1\left(\Delta \eta_{2}=4.2\right)$. The central activity is determined using tracks in the range of $-0.9<\eta<0.9$ $\left(\Delta \eta_{\text {signal }}=1.8\right)$. In this analysis, a total of $3 \times 10^{7} \mathrm{MB}_{\mathrm{OR}}$ events at $\sqrt{s}=7 \mathrm{TeV}$ collected in 2010 are used. Only events with a vertex within $\pm 4 \mathrm{~cm}$ from the nominal vertex position in the direction of the beam line are accepted. For each run the gap-fraction is calculated. Runs where this fraction has a deviation of more than $3 \sigma$ from the data taking period mean are rejected. The double-gap fraction is found to be $7.63 \pm 0.02$ (stat) \pm 0.87 (syst) $\times 10^{-4}$ [7]. The fraction of the background in the double gap topology is found to be about $10 \%$ and was estimated by studying the contribution of NSD, SD and DD processes using PHOJET and PYTHIA6. However, a tuning is needed since both generators are unable to describe the double gap fraction observed in ALICE data. This analysis is ongoing.

\section{Summary}

- Cross-sections for inelastic, single- and double diffraction were measured by ALICE at LHC energies.

- ALICE SD and DD data agree with UA5 at $\sqrt{s}=900$ $\mathrm{GeV} / c^{2}$.
- Two sides of detector sensitive to different $M_{X}$ values, give the same result for SD, as they should.

- When changing from $M_{X}<200 \mathrm{GeV} / c^{2}$ to $M_{X}<100$ $\mathrm{GeV} / c^{2}$ or $M_{X}<50 \mathrm{GeV} / c^{2}$, data follow the model [1].

- Tuned PYTHIA and PHOJET reproduce extremely well measured relative trigger rates at the 3 energies.

\section{References}

[1] A.B. Kaidalov, M.G. Poghosyan, in Proceedings of the 13th International Conference on Elastic and Diffractive Scattering ( "Blois Worshop”), ed. by M. Deile, D. d'Enterria, A. De Roeck, CERN, 2009 (DESY, Hamburg, 2010). arXiv:0909.5156 [hep-ph]

[2] B. Abelev et al. (ALICE Collaboration), Eur. Phys. J.C 73, 2456 (2013)

[3] K. Aamodt et al. (ALICE Collaboration), JINST 3, S08002 (2008).

[4] G. Aad et al. (ATLAS Collaboration), Nat. Commun. 2, 463 (2011)

[5] CMS Collaboration, Performance Analysis Note CMS-PAS-FWD11-001

[6] G. Antchev et al. (TOTEM Collaboration), Europhys. Lett. 96, 21002 (2011)

[7] Felix Reidt, arXiv:1301.3507v1 [hep-ex]

[8] R.E. Ansorge et al. (UA5 Collaboration), Z. Phys. C 33, 175 (1986)

[9] G.J. Alner et al. (UA5 Collaboration), Z. Phys. C 33, 153 (1986)

[10] E. Gotsman, E. Levin, U. Maor, Phys. Lett. B, in press. arXiv: 1208.0898 [hep-ph]

[11] K. Goulianos, Phys. Rev. D 80, 111901(R) (2009)

K. Goulianos, in Proceedings of the 13th International Conference on Elastic and Diffractive Scattering ("Blois Worshop”), ed. by M. Deile, D.d' Enterria, A. De Roeck, CERN, 2009 (DESY, Hamburg, 2010). arXiv: 1002.3527

K. Goulianos, in Proceedings of Forward Physics at LHC, Workshop, La Biodola, Elba (2010), p. 17. arXiv:1009.5413 [hep-ph]

K. Goulianos, arXiv:1012.5169 [hep-ex]

$\mathrm{K}$. Goulianos, in Proceedings of the 46th Rencontres de Moriond, La Thuile (2011). arXiv:1105.4916 [hep-ph]

[12] S. Ostapchenko, Phys. Rev. D 77, 034009 (2008)

S. Ostapchenko, Phys. Rev. D 81, 114028 (2010)

S. Ostapchenko, Phys. Rev. D 83, 114018 (2011)

[13] M.G. Ryskin, A.D. Martin, V. Khoze, Eur. Phys. J. C 54, 199 (2008)

M.G. Ryskin, A.D. Martin, V. Khoze, Eur. Phys. J. C 60, 249 (2009) M.G. Ryskin, A.D. Martin, V. Khoze, Eur. Phys. J. C 71, 1617 (2011) 